\title{
Fatores associados ao uso de psicofármacos em idosos asilados
}

\author{
Factors associated with the use of psychoactive drugs in institutionalized elderly
}

\author{
Giancarlo Lucchetti ${ }^{1}$, Alessandra Lamas Granero ${ }^{2}$, Sueli Luciano Pires ${ }^{3}$, Milton Luiz Gorzoni ${ }^{4}$, Sérgio \\ Tamai $^{5}$
}

${ }^{1}$ Médico. Especialista em Clínica Médica. Especializando em Geriatria, Irmandade da Santa Casa de Misericórdia de São Paulo, São Paulo, SP. ${ }^{2}$ Médica, Faculdade de Ciências Médicas, Santa Casa de São Paulo, São Paulo, SP. Especializanda em Geriatria, Centro Interdisciplinar de Assistência e Pesquisa em Envelhecimento (CIAPE), Belo Horizonte, MG. ${ }^{3}$ Especialista em Geriatria, Sociedade Brasileira de Geriatria e Gerontologia. Doutoranda, Irmandade da Santa Casa de Misericórdia de São Paulo. Diretora técnica, Hospital Geriátrico e de Convalescentes Dom Pedro II, São Paulo, SP. ${ }^{4}$ Especialista em Geriatria, Sociedade Brasileira de Geriatria e Gerontologia. Doutor, Irmandade da Santa Casa de Misericórdia de São Paulo. Coordenador, Serviço de Geriatria, Irmandade da Santa Casa de Misericórdia de São Paulo. ${ }^{5}$ Especialista em Psiquiatria. Doutor, Psiquiatria, Universidade de São Paulo, São Paulo, SP. Chefe, Departamento de Psiquiatria e Psicologia Médica, Faculdade de Ciências Médicas, Santa Casa de São Paulo.

\section{Resumo}

Introdução: As instituições de longa permanência para idosos (ILPI) apresentam altas prevalências de internados em uso de psicofármacos para o controle de distúrbios comportamentais. Há, porém, poucos estudos brasileiros sobre esse aspecto de abordagem terapêutica em ILPI.

Objetivo: Avaliar os fatores associados ao uso de psicofármacos em pacientes idosos institucionalizados.

Métodos: Foi realizado estudo transversal e retrospectivo por meio de análise de prontuários de todos os idosos (60 anos ou mais) internados em um ILPI, independentemente das doenças apresentadas. Regressão logística foi realizada para verificar os fatores associados ao uso de psicofármacos na instituição.

Resultados: Foram avaliados 209 pacientes (73,2\% mulheres), predominantemente portadores de síndromes demenciais, sequelas de acidentes vasculares cerebrais ou de traumas de crânio e hipertensão arterial sistêmica. Os psicofármacos estavam prescritos para 123 internados (58,9\% do total), principalmente neurolépticos e antidepressivos. Após a regressão logística, encontrou-se associação entre a prescrição de neurolépticos e demência $(p=0,000)$, enquanto que o uso de antidepressivos esteve associado a maior número de medicamentos $(p=0,004)$ e presença de depressão $(p=0,000)$. O uso de psicofármacos em geral esteve fortemente associado com depressão $(p=0,000)$, presença de demência $(p=0,006)$ e presença de doenças psiquiátricas $(\mathrm{p}=0,02)$. Não houve associação com as variáveis sexo, funcionalidade e idade.

Conclusão: Há alto consumo de psicofármacos em ILPI. A associação destes com polifarmácia e depressão é significativa, e os portadores de demência foram os que mais fizeram uso dos neurolépticos. Fatores como idade e sexo, normalmente relevantes em pacientes ambulatoriais, não apresentaram associação em institucionalizados.

Descritores: Idosos, drogas psicoativas, antipsicóticos, instituição de longa permanência para idosos.

\begin{abstract}
Introduction: Nursing homes providing elderly care present a high prevalence of interns using psychoactive drugs for the control of behavioral disorders. Few Brazilian studies have so far focused on this aspect of elderly care.

Objective: To evaluate the factors associated with use of psychoactive drugs in institutionalized elderly patients.

Methods: A cross-sectional, retrospective study was carried out based on medical charts of all elderly patients (60 years and over) treated in a nursing home, independently of their diseases. Logistic regression analysis was performed to assess factors associated with the use of psychoactive drugs in the institution. Results: Two hundred and nine patients were evaluated (73.2\% females), suffering predominantly of dementia syndromes, sequelae of stroke or cranial trauma, and hypertension. Of these, 123 patients (58.9\%) were using some type of psychoactive drug, mainly antipsychotics and antidepressants. Logistic regression analysis revealed an association between antipsychotic drug use and dementia $(p=0.000)$, whereas antidepressant drug use was associated with a higher number of drugs $(p=0.004)$ and depression $(p=0.000)$. In general, the use of psychoactive drugs was strongly associated with depression $(p=0.000)$, dementia $(p=0.006)$, and psychiatric disorders $(p=0.02)$. There were no associations with sex, functional status or age.

Conclusion: There is a high consumption of psychoactive drugs in nursing homes. The association between polypharmacy and depression is evident, and patients with dementia were shown to receive more antipsychotics than other patients. Factors such as age or sex, usually relevant among outpatients, did not present association with psychoactive drug use in nursing home patients.
\end{abstract}

Keywords: Elderly, psychoactive drugs, antipsychotics, nursing homes.

Correspondência:

Giancarlo Lucchetti, Av. Juriti, 367 apto. 131, Moema, CEP 04520-000, São Paulo, SP. Tel.: (11) 5052-1298. E-mail: g.lucchetti@yahoo.com.br

Não foram declarados conflitos de interesse associados à publicação deste artigo.

Copyright $\odot$ Revista de Psiquiatria do Rio Grande do Sul - APRS

Recebido em 12/08/2009. Aceito em 14/01/2010. 


\section{Introdução}

Uma significativa parcela dos medicamentos prescritos no Brasil é da classe dos psicofármacos. Estima-se que pelo menos $13 \%$ do total de fármacos consumidos em nosso país envolva benzodiazepínicos, antidepressivos, neurolépticos, anticonvulsivantes ou estimulantes do sistema nervoso central ${ }^{1}$.

Os pacientes internados em instituições de longa permanência para idosos (ILPI) merecem atenção especial quanto ao consumo desses grupos medicamentosos, visto seu uso corriqueiro em quadros demenciais, depressões e distúrbios comportamentais. Estima-se que a prevalência de psicofármacos em asilados chegue a $63,0 \% \%^{2}$, sendo esses medicamentos usualmente prescritos por médicos não psiquiatras em decorrência da necessidade de controle comportamental, presença de depressão e transtornos do sono. Estudos recentes apontam para uma maior taxa de mortalidade entre idosos dementados que recebem neurolépticos ${ }^{3,4}$.

Apesar de os institucionalizados já corresponderem a $1,5 \%$ da população brasileira ${ }^{5}$, há poucos estudos sobre prescrição de psicofármacos e os fatores relacionados à sua indicação nessa população. Na literatura brasileira consultada, encontrou-se um único estudo que abordou essa temática em ILPI, realizado em Rio Claro (SP) ${ }^{2}$.

O presente estudo tem como objetivo traçar o perfil dos medicamentos psicoativos em uso em um ILPI e identificar os fatores associados ao uso de diversos grupos desses medicamentos.

\section{Casuística e método}

\section{Tipo de estudo e amostra}

Foi realizado um estudo transversal, retrospectivo, por meio de análise de prontuários de todos os idosos (idade igual ou maior que 60 anos) internados no Hospital Geriátrico e de Convalescentes Dom Pedro II, conforme o censo hospitalar datado de 7 de fevereiro de 2005, independentemente das doenças apresentadas. Essa instituição pertence à Irmandade da Santa Casa de Misericórdia de São Paulo e possui 505 leitos para ambos os sexos. Dos 505 internados, 197 foram excluídos devido a idade menor de 60 anos, 15 pela internação na unidade de agudos (pneumonias, infecções do trato urinário, dentre outras), 81 devido à falta de dados durante a coleta e 3 por óbito, constituindo-se a amostra final de 209 pacientes.

\section{Procedimentos}

Elaborou-se um protocolo constituído de dados sociodemográficos, número e tipos de doenças, número de medicamentos, presença de polifarmácia (definida no presente estudo como mais de cinco medicamentos por paciente), tipos de medicamentos psicoativos usados (divididos por grupos: benzodiazepínicos, antidepressivos, neurolépticos típicos ou atípicos e anticonvulsivantes) e dependência funcional pela escala de $\mathrm{Katz}^{6}$.

\section{Análise estatística}

As variáveis qualitativas foram analisadas mediante análise descritiva, e as quantitativas, mediante medidas resumo. Em seguida, foi realizada análise bivariada por meio do teste do qui-quadrado, para explorar a magnitude de associações (razões de prevalência) entre as variáveis independentes e dependentes. As seguintes variáveis dependentes foram utilizadas: uso de neurolépticos ( $\operatorname{sim} /$ não), uso de antidepressivos ( $\operatorname{sim} /$ não), uso de benzodiazepínicos ( $\operatorname{sim} /$ não) e uso total de psicofármacos ( $\operatorname{sim} /$ não). Como variáveis independentes, foram utilizadas idade, funcionalidade (pela escala de Katz), sexo, demência, sequela de acidente vascular cerebral ou trauma cranioencefálico, número de medicamentos, depressão, número de diagnósticos, doenças psiquiátricas e medicamentos cardiovasculares.

Foi conduzida então a regressão logística, no intuito de controlar fatores de confundimento e fatores de interação e predizer quais variáveis eram significantes dentre as escolhidas. Foram realizados os testes de Omnibus para os coeficientes do modelo e de Hosmer-Lemeshow no intuito de rejeitar a hipótese nula (neste caso, de que não haveria preditores para as variáveis dependentes), de forma a verificar a qualidade de ajuste do modelo. As variáveis independentes e dependentes foram as mesmas utilizadas na análise bivariada. $\mathrm{O}$ estudo considerou $\mathrm{p}<0,05$ como significante e nível de significância de 95,0\%. A análise estatística foi realizada utilizando o programa Statistical Package for the Social Sciences (SPSS), versão 17.

\section{Comitê de ética}

O presente estudo foi enviado e aprovado pelo Comitê de Ética em Pesquisa da Santa Casa de São Paulo (protocolo $\left.n^{\circ} 308 / 05\right)$.

\section{Resultados}

A casuística final constituiu-se de 209 pacientes, sendo 153 mulheres $(73,2 \%)$, com idade média de 75,9 anos $(65,2-$ $85,6)$ e tempo médio de institucionalização de 95,3 meses $(63,3-127,3)$

As principais doenças encontradas foram: hipertensão arterial sistêmica em 142 internados (67,9\%); síndromes demenciais em 77 (36,8\%); sequelas de trauma cranioencefálico ou acidente vascular cerebral em 71 (33,97\%); e doenças cardiovasculares em 70 pacientes $(33,4 \%)$. A depressão esteve presente em 33 pacientes $(15,8 \%)$, e as doenças 
psiquiátricas (esquizofrenia, transtorno afetivo-bipolar, transtorno obsessivo-compulsivo) em 23 (11,0\%).

Os psicofármacos estavam prescritos para 123 internados (58,9\% do total): 69 pacientes $(33,0 \%)$ usavam neurolépticos (desses, 78,0\% eram atípicos, em sua maioria risperidona, e $22,0 \%$ eram típicos, em sua maioria haloperidol - ambos eram os medicamentos padronizados pela instituição); 37 (17,7\%) usavam antidepressivos (fluoxetina, sertralina, amitriptilina, nortriptilina, citalopram, paroxetina e imipramina); 28 (13,4\%) benzodiazepínicos (diazepam, clonazepam e lorazepam); e 25 pacientes $(12,0 \%)$ usavam anticonvulsivantes.

A divisão dos pacientes em subgrupos pode ser melhor visualizada na Tabela 1. A Tabela 2 apresenta os resultados da análise bivariada. A regressão logística encontrou as seguintes associações (Tabela 3):

- Uso de neurolépticos: teste de Omnibus/qui-quadrado $=26,6(p=0,003)$; teste de Hosmer-Lemeshow/qui-quadrado $=6,00(p=0,647)$. Associado fortemente a presença de demência $(p=0,000)$.
- Uso de benzodiazepínicos: teste de Omnibus/qui-quadrado $=25,63(\mathrm{p}=0,004)$; teste de Hosmer-Lemeshow/quiquadrado $=12,3(\mathrm{p}=0,138)$. Associado fortemente a doenças psiquiátricas $(\mathrm{p}=0,004)$ e moderadamente a maior número de medicamentos $(\mathrm{p}=0,017)$.

- Uso de antidepressivos: teste de Omnibus/qui-quadrado $=71,52(\mathrm{p}=0,000)$; teste de Hosmer-Lemeshow/qui-quadrado $=4,85(p=0,773)$. Associado fortemente a presença de depressão $(p=0,000)$ e fortemente a maior número de medicamentos $(\mathrm{p}=0,004)$.

- Uso de medicamentos psicoativos em geral: teste de Omnibus/qui-quadrado $=26,65(\mathrm{p}=0,003)$; teste de Hosmer-Lemeshow/qui-quadrado $=6,00(p=0,647)$. Associado fortemente a presença de depressão $(p=0,000)$, fortemente a presença de demência $(\mathrm{p}=0,006)$ e de forma fraca a presença de doenças psiquiátricas $(\mathrm{p}=0,02)$.

- Não houve associação estatisticamente significante com as variáveis idade, sexo e funcionalidade para nenhum grupo de medicamentos.

Tabela 1 - Porcentagem das diferentes classes de psicofármacos nos subgrupos portadores de demência, sequela de AVC/TCE, depressão, doenças psiquiátricas e pacientes sem indicações óbvias de psicofármacos e no total de pacientes asilados no Hospital Geriátrico e de Convalescentes Dom Pedro II, São Paulo (SP)

\begin{tabular}{|c|c|c|c|c|}
\hline & $\begin{array}{c}\text { Psicoativos } \\
\text { n (\%) }\end{array}$ & $\begin{array}{c}\text { Neurolépticos } \\
\text { n (\%) }\end{array}$ & $\begin{array}{c}\text { Benzodiazepínicos } \\
\text { n (\%) }\end{array}$ & $\begin{array}{c}\text { Antidepressivos } \\
\text { n (\%) }\end{array}$ \\
\hline Demência $(n=77)$ & $52(67,5)$ & $44(57,1)$ & $8(10,4)$ & $7(9,1)$ \\
\hline Sequela AVC/TCE $(n=71)$ & $40(56,3)$ & $16(22,5)$ & $5(7,0)$ & $14(19,7)$ \\
\hline Depressão $(n=33)$ & $27(81,8)$ & $4(12,1)$ & $5(15,2)$ & $24(72,7)$ \\
\hline Doenças psiquiátricas $(\mathrm{n}=23)$ & $17(73,9)$ & $11(47,8)$ & $9(39,1)$ & $4(17,4)$ \\
\hline Pacientes sem indicações óbvias de psicofármacos* $(n=100)$ & $54(54,0)$ & $39(39,0)$ & $10(10,0)$ & $8(8,0)$ \\
\hline Todos os pacientes $(\mathrm{n}=209)$ & $123(58,9)$ & $69(33,0)$ & $28(13,4)$ & $37(17,7)$ \\
\hline
\end{tabular}

AVC/TCE = acidente vascular cerebral/trauma cranioencefálico.

* Amostra de pacientes com exclusão daqueles com depressão (em uso presumido de antidepressivos), com sequelas de AVC/TCE (uso presumido de anticonvulsivantes) e com transtornos psiquiátricos (uso presumido de medicamentos psiquiátricos).

\section{Discussão}

Há aproximadamente 6 mil ILPI no Brasil, com mais de 100 mil asilados ${ }^{7}$. O padrão dos moradores dessas instituições varia de residentes hígidos até portadores de comorbidades como neoplasias, doenças psiquiátricas, síndromes demenciais, sequelas de traumas e acidente cerebrovascular e síndrome do imobilismo ${ }^{6}$.

Torna-se, assim, rotineira a necessidade da prescrição de psicofármacos visando ao controle de sintomas como agitação, agressividade, delírios, depressão e ansiedade. Muitas vezes, o prescritor não psiquiatra não necessariamente apresenta familiaridade com o perfil farmacológico desses medicamentos e seus possíveis efeitos colaterais e interações medicamentosas ${ }^{8}$.
O presente estudo observou que o perfil do hospital avaliado é composto predominantemente por mulheres na faixa dos 70 a 80 anos, dado compatível com as casuísticas de outros estudos brasileiros envolvendo asilados ${ }^{6,9}$. A prescrição de psicofármacos em $58,9 \%$ dos internados é próxima da relatada por Stella et al. em Rio Claro $(63,1 \%)^{2}$. A literatura internacional consultada apresentou percentuais entre $46,8 \mathrm{e}$ $83,0 \%$ de institucionalizados em uso de medicamentos psicoativos $^{10-15}$. Essa diferença de valores pode ser justificada pela modalidade de assistência (independente ou não) nas diferentes ILPI e pelo tipo e gravidade das doenças.

$\mathrm{O}$ uso de medicamentos psicoativos no contexto das IPLI pode ser justificado pelas características clínicas dos pacientes, que possuem síndromes demenciais avançadas cursando com agressividade, agitação, doenças psiquiátricas 
Uso de psicofármacos em asilados - LucChETTI ET AL.

Tabela 2 - Análise bivariada dos fatores associados ao uso de psicofármacos em asilados no Hospital Geriátrico e de Convalescentes Dom Pedro II, São Paulo (SP)

\begin{tabular}{|c|c|c|c|c|c|c|c|c|}
\hline \multirow[b]{2}{*}{ Fator } & \multicolumn{2}{|c|}{$\begin{array}{l}\text { Benzodiazepínicos } \\
\quad(\mathrm{n}=\mathbf{2 8})\end{array}$} & \multicolumn{2}{|c|}{$\begin{array}{l}\text { Antidepressivos } \\
\qquad(\mathrm{n}=\mathbf{3 7})\end{array}$} & \multicolumn{2}{|c|}{$\begin{array}{c}\text { Total de } \\
\text { neurolépticos } \\
(\mathrm{n}=69)\end{array}$} & \multicolumn{2}{|c|}{$\begin{array}{c}\text { Total de } \\
\text { psicofármacos } \\
(\mathrm{n}=\mathbf{1 2 3})\end{array}$} \\
\hline & Sim (\%) & Não (\%) & Sim (\%) & Não (\%) & Sim (\%) & Não (\%) & Sim (\%) & Não (\%) \\
\hline Idade & & $\mathrm{p}=0,27$ & & $\mathrm{p}=0,71$ & & $\mathrm{p}=0,98$ & & $\mathrm{p}=0,31$ \\
\hline 60 a 79 anos & 71,4 & 60,8 & 64,9 & 61,6 & 62,3 & 62,1 & 65 & 58,1 \\
\hline 80 a 120 anos & 28,6 & 39,2 & 35,1 & 38,4 & 37,7 & 37,9 & 35 & 41,9 \\
\hline Funcionalidade & & $\mathrm{p}=0,22$ & & $\mathrm{p}=0,76$ & & $\mathrm{p}=0,99$ & & $\mathrm{p}=0,75$ \\
\hline Independente & 42,9 & 55,2 & 51,4 & 54,1 & 53,6 & 53,6 & 54,5 & 52,3 \\
\hline Dependente & 57,1 & 44,8 & 48,6 & 45,9 & 46,4 & 46,4 & 45,5 & 47,7 \\
\hline Sexo & & $\mathrm{p}=0,81$ & & $\mathrm{p}=0,7$ & & $\mathrm{p}=0,4$ & & $\mathrm{p}=0,33$ \\
\hline Masculino & 25 & 27,1 & 24,3 & 27,3 & 23,2 & 28,6 & 29,3 & 23,3 \\
\hline Feminino & 75 & 72,9 & 75,7 & 72,7 & 76,8 & 71,4 & 70,7 & 76,7 \\
\hline Demência & & $\mathrm{p}=0,32$ & & $\mathrm{p}=0,01^{*}$ & & $\mathrm{p}=0,00^{*}$ & & $\mathrm{p}=0,05$ \\
\hline Presente & 28,6 & 38,1 & 18,9 & 40,7 & 63,8 & 23,6 & 42,3 & 29,1 \\
\hline Ausente & 71,4 & 61,9 & 81,1 & 59,3 & 36,2 & 76,4 & 57,7 & 70,9 \\
\hline Sequela AVC/TCE & & $\mathrm{p}=0,05$ & & $\mathrm{p}=0,58$ & & $\mathrm{p}=0,02^{*}$ & & $\mathrm{p}=0,59$ \\
\hline Presente & 17,9 & 36,5 & 37,8 & 33,1 & 23,2 & 39,3 & 32,5 & 36 \\
\hline Ausente & 82,1 & 63,5 & 62,2 & 66,9 & 76,8 & 60,7 & 67,5 & 54 \\
\hline Medicamentos & & $\mathrm{p}=0,01 *$ & & $\mathrm{p}=0,03^{*}$ & & $\mathrm{p}=0,07$ & & $\mathrm{p}=0,55$ \\
\hline 0 a 4 & 32,1 & 56,9 & 37,8 & 57 & 62,3 & 49,3 & 55,3 & 51,2 \\
\hline Mais de 5 & 67,9 & 43,1 & 62,2 & 43 & 37,7 & 50,7 & 44,7 & 48,8 \\
\hline Depressão & & $\mathrm{p}=0,74$ & & $\mathrm{p}=0,00^{*}$ & & $\mathrm{p}=0,00^{*}$ & & $\mathrm{p}=0,00^{*}$ \\
\hline Presente & 17,9 & 15,5 & 64,9 & 5,2 & 5,8 & 20,7 & 22 & 7 \\
\hline Ausente & 82,1 & 84,5 & 35,1 & 94,8 & 94,2 & 79,3 & 78 & 93 \\
\hline Número de diagnósticos & & $\mathrm{p}=0,55$ & & $\mathrm{p}=0,00^{*}$ & & $\mathrm{p}=0,00^{*}$ & & $\mathrm{p}=0,40$ \\
\hline 0 a 3 & 28,6 & 34,3 & 13,5 & 37,8 & 46,4 & 27,1 & 35,8 & 30,2 \\
\hline Acima de 3 & 71,4 & 65,7 & 86,5 & 62,2 & 53,6 & 72,9 & 64,2 & 69,8 \\
\hline Doenças psiquiátricas & & $\mathrm{p}=0,00^{*}$ & & $\mathrm{p}=0,96$ & & $\mathrm{p}=0,10$ & & $\mathrm{p}=0,11$ \\
\hline Presente & 32,1 & 7,7 & 10,8 & 11 & 15,9 & 8,6 & 13,8 & 7 \\
\hline Ausente & 67,9 & 92,3 & 89,2 & 89 & 84,1 & 91,4 & 86,2 & 93 \\
\hline Medicamentos cardiovasculares & & $\mathrm{p}=0,45$ & & $\mathrm{p}=0,43$ & & $\mathrm{p}=0,00^{*}$ & & $\mathrm{p}=0,04^{*}$ \\
\hline Presentes & 64,3 & 71,3 & 75,7 & 69,2 & 58 & 76,4 & 65 & 77,9 \\
\hline Ausentes & 35,7 & 28,7 & 24,3 & 30,8 & 42 & 23,6 & 35 & 22,1 \\
\hline
\end{tabular}

AVC/TCE $=$ acidente vascular cerebral/trauma cranioencefálico.

* Estatisticamente significante.

Tabela 3 - Coeficientes de regressão do modelo de regressão logística relacionando uso de benzodiazepínicos, antidepressivos, neurolépticos e psicofármacos em geral com variáveis independentes diversas

\begin{tabular}{|c|c|c|c|c|c|c|c|c|}
\hline & \multicolumn{2}{|c|}{ Benzodiazepínicos } & \multicolumn{2}{|c|}{ Antidepressivos } & \multicolumn{2}{|c|}{ Neurolépticos } & \multicolumn{2}{|c|}{ Total de psicofármacos } \\
\hline & Beta $(\beta)$ & $\mathbf{p}$ & Beta $(\beta)$ & $\mathbf{p}$ & Beta $(\beta)$ & $\mathbf{p}$ & Beta $(\beta)$ & $\mathbf{p}$ \\
\hline Sexo masculino & $-0,029$ & 0,957 & 0,290 & 0,633 & $-0,424$ & 0,314 & $-0,143$ & 0,700 \\
\hline Idade (anos) & 0,018 & 0,477 & $-0,006$ & 0,837 & 0,031 & 0,113 & 0,027 & 0,122 \\
\hline Maior dependência funcional & $-0,064$ & 0,518 & $-0,026$ & 0,813 & 0,057 & 0,453 & 0,038 & 0,582 \\
\hline Presença de demência & 0,184 & 0,728 & $-0,420$ & 0,501 & 2,063 & $0,000 *$ & 1,013 & $0,006 *$ \\
\hline Presença de sequela de AVC/TCE & $-0,418$ & 0,486 & $-0,073$ & 0,902 & $-0,365$ & 0,382 & $-0,354$ & 0,334 \\
\hline Presença de depressão & 0,580 & 0,329 & 3,875 & $0,000 *$ & $-1,025$ & 0,085 & 2,011 & $0,000 *$ \\
\hline Ausência de polifarmácia & $-1,320$ & $0,017 *$ & $-1,146$ & $0,036 *$ & $-0,179$ & 0,644 & $-0,167$ & 0,847 \\
\hline Número de diagnósticos & 0,038 & 0,792 & 0,054 & 0,716 & 0,091 & 0,407 & 0,116 & 0,208 \\
\hline Doenças psiquiátricas & 1,614 & $0,004 *$ & 0,675 & 0,380 & 1,056 & 0,044 & 1,258 & $0,020 *$ \\
\hline Medicamentos cardiovasculares & $-0,738$ & 0,190 & $-0,914$ & 0,161 & $-0,754$ & 0,062 & $-0,614$ & 0,101 \\
\hline Tempo de institucionalização & $-0,550$ & 0,124 & 0,231 & 0,438 & $-0,159$ & 0,472 & $-0,173$ & 0,371 \\
\hline Constante & 0,920 & 0,775 & $-3,029$ & 0,406 & $-1,715$ & 0,512 & $-7,493$ & 0,002 \\
\hline
\end{tabular}

$\mathrm{AVC} / \mathrm{TCE}=$ acidente vascular cerebral/trauma cranioencefálico.

$* \mathrm{p}<0,05$ 
de difícil controle, isolamento social, ambiente desconhecido, quadros depressivos, quadros ansiosos, má qualidade do sono, perda da autonomia e dependência física.

$\mathrm{O}$ encontro frequente de prescrições com neurolépticos também foi observado em outros estudos ${ }^{16,17}$, havendo predomínio do uso de neurolépticos atípicos, como observado na presente casuística. A relação entre demência e consumo de neurolépticos, fato usual em ILPI, é dado relatado com constância ${ }^{18-21}$. Estudos demonstram que os institucionalizados cursam com maior prevalência de depressão, levando a uma maior utilização de antidepressivos, podendo chegar a $31,0 \%$ em algumas casuísticas, número maior se comparado ao presente estudo ${ }^{20,22}$. Deve-se, porém, enfatizar que o encontro de $17,7 \%$ de prescrições com fármacos antidepressivos nesta casuística não deve ser considerado como menor, visto que praticamente um em cada cinco institucionalizados utilizava esse grupo medicamentoso. O quadro depressivo é achado comum nas ILPI devido a fatores como as limitações físicas e a dependência funcional, associadas ao isolamento e à negação no intuito de diminuir a percepção de um ambiente que não lhe é conhecido ${ }^{23}$.

Igualmente, apesar dos conhecidos riscos e efeitos colaterais que os benzodiazepínicos provocam em idosos, tornase muitas vezes difícil sua retirada em quadros de ansiedade e distúrbios do sono, provocando seu consumo em um percentual significativo de asilados ${ }^{8,24}$. O paciente asilado muitas vezes possui qualidade de sono ruim devido a aspectos como a iluminação local, barulhos e interrupções da equipe de enfermagem, além de maior prevalência de incontinência urinária nessa população ${ }^{25}$.

Quanto aos fatores associados ao uso dos medicamentos psicoativos, o uso de neurolépticos em geral foi relacionado à presença de demência. Esse achado é compatível com outros estudos ${ }^{18,20}$, que relataram que o controle comportamental (medicamentoso ou não) encontra-se entre as principais preocupações da equipe de profissionais vinculada a ILPI, em que os internados se encontram em estágios mais avançados e de difícil manejo do quadro demencial ${ }^{26}$. Apesar de, na análise bivariada, o menor número de medicamentos ter sido correlato ao uso de neurolépticos (o que poderia ser justificado pela possibilidade terapêutica nesse estágio da doença restringir-se aos distúrbios comportamentais ${ }^{27}$ ), essa relação não foi observada na regressão logística.

O uso de benzodiazepínicos mostrou-se associado com polifarmácia, o que pode ser justificado pelas características dos pacientes residentes em ILPI (várias doenças simultâneas e incapacitantes), os quais, para controle comportamental e/ou de distúrbios do sono, muitas vezes necessitam de vários medicamentos, incluindo, eventualmente, benzodiazepínicos. Considerando-se, porém, a dinâmica de uma ILPI, o número de pacientes em uso de benzodiazepínicos foi menor do que em outro estudo realizado em um ambulatório de Porto Alegre ${ }^{28}$, o que pode ser justificado pelo caráter de ensino (revisões periódicas de prontuários e prescrições) apresentado pela instituição avaliada no presente estudo. O uso de benzodiazepínicos se associou ainda à presença de doenças psiquiátricas, um achado compatível com o fato de esse grupo de pacientes ser de difícil manejo e necessitar de vários medicamentos simultaneamente.

Correlacionou-se o consumo de antidepressivos com depressão (fato esperado) e polifarmácia, demonstrando a importância do uso racional de medicamentos pelo idoso.

$\mathrm{O}$ uso de medicamentos psicoativos em geral demonstrou associação com a presença de depressão, achado compatível com estudos internacionais ${ }^{26}$, sugerindo a importância desse quadro entre internados em ILPI e o caráter da muitas vezes necessária associação de mais de um medicamento para seu tratamento, principalmente nos casos correlacionados também a transtornos psiquiátricos, demência e sequelas neurológicas.

Não se encontrou associação entre medicamentos psicoativos e faixa etária no presente estudo, achado concordante com alguns estudos ${ }^{29,30}$ porém divergente de outros ${ }^{2,15}$. O fato de a presente casuística ser composta apenas de idosos pode ser uma das justificativas para esse achado.

Quanto ao sexo, não foi achada nenhuma relação, dado compatível com alguns estudos em ILPI ${ }^{31}$ porém divergente de outros que mostraram relação com o sexo feminino ${ }^{2,32} \mathrm{e}$ com o sexo masculino ${ }^{33}$. Ou seja, o sexo ainda não é um fator certamente relacionado ao uso de medicamentos psicoativos em ILPI.

A funcionalidade, importante no contexto da ILPI, não foi preditora do uso de medicamentos psicoativos, dado este já referido em estudo anterior ${ }^{34}$.

O presente estudo diferiu do único estudo nacional encontrado na literatura consultada que avaliou os fatores relacionados a ILPI no Brasil, realizado em Rio Claro ${ }^{2}$. Crê-se que a razão para isso foi que a presente casuística é composta de pacientes com síndromes demenciais e doenças cerebrovasculares, enquanto que Stella et al. ${ }^{2}$ avaliaram internados com preponderância de doenças cardiovasculares. Relações de sexo e idade não foram evidenciadas em estudos de casuísticas originárias de $\mathrm{ILPI}^{31}$, sugerindo que essas relações se tornam mais evidentes fora do contexto de asilados em geral.

Algumas limitações são inerentes ao presente estudo. A primeira refere-se ao desenho do estudo (retrospectivo, por meio de análise de prontuários): durante a coleta de dados, houve uma perda amostral de aproximadamente $26 \%$ dos idosos institucionalizados. A segunda limitação diz respeito ao fato de esta ser uma análise de uma única instituição, que, apesar de ser de grande porte, possui suas próprias peculiaridades. Em terceiro lugar, trata-se de uma instituição em que a maioria dos asilados possui moderada ou alta dependência funcional. Sendo assim, mostra-se necessária a replicação de novos estudos nacionais com a mesma temática para que se possa concluir quais são os fatores mais importantes associados ao uso de medicamentos psicoativos em asilados brasileiros. 


\section{Conclusão}

Há um alto consumo de psicofármacos em ILPI, sua associação com polifarmácia e depressão é significativa, e os portadores de demência foram os que mais fizeram uso de neurolépticos. Levando-se em consideração que estudos recentes apontam para uma maior mortalidade desses pacientes em uso de neurolépticos, especialmente os atípicos, é necessário que novos estudos avaliem a relação risco-benefício do uso dessas medicações.

Fatores como idade e sexo, comuns no contexto ambulatorial, não apresentaram associação nos institucionalizados.

\section{Referências}

1. National Disease and Therapeutic Index - IMS Health; December 2000 [Internet] [accessed 2009 Jun 18]. Available from: https://web01.imshealth.com/ndti/ndtilogin.aspx

2. Stella F, Caetano D, Pacheco JL, Sé EVG, Lacerda ALT. Factors influencing psychotropic prescription by non-psychiatrist physicians in a nursing home for the elderly in Brazil. Sao Paulo Med J. 2006;124(5):253-6.

3. Schneider LS, Dagerman KS, Insel P. Risk of death with atypical antipsychotic drug treatment for dementia: meta-analysis of randomized placebo-controlled trials. JAMA. 2005;294:1934-43.

4. Ballard C, Hanney ML, Theodoulou M, Douglas S, McShane R, Kossakowski K, et al. The dementia antipsychotic withdrawal trial (DART-AD): long-term follow-up of a randomised placebo-controlled trial. Lancet Neurol. 2009;8(2):151-7.

5. Camarano AA. Brazilian population ageing: differences in well-being by rural and urban areas [Internet]. Geneva: United Nations; 2002 [accessed 2009 May 24; 33 screens]. Available from: http://www.ipea.gov.br

6. Camarano AA. Instituições de longa permanência e outras modalidades de arranjos domiciliares para idosos. In: Néri AL, org. Idosos no Brasil: vivência, desafios e expectativas na terceira idade. São Paulo: Fundação Perseu Abramo, 2007. p. 1-288.

7. Araújo MOPH, Ceolim MF. Avaliação do grau de independência de idosos residentes em instituições de longa permanência. Rev Esc Enferm USP. 2007;41(3):378-85.

8. Orlandi P, Noto AR. Uso indevido de benzodiazepínicos: um estudo com informanteschave no município de São Paulo. Rev Latinoam Enferm. 2005;13(especial):896-902.

9. Chaimowicz F, Greco DB. Dinâmica da institucionalização de idosos em Belo Horizonte, Brasil. Rev Saude Publica. 1999;33(5):454-60.

10. Mamun K, Goh-Tan CYE, Ng LL. Prescribing psychoactive medications in nursing homes: current practice in Singapore. Singapore Med J. 2003;12:625-9.

11 van Dijk KN, de Vries CS, van den Berg PB, Brouwers JRBJ, de Jong-van den Berg LTW. Drug utilisation in Dutch nursing homes. Eur J Clin Pharmacol. 2000;55(10):765-71.

12. Taylor LF. Psychotropic drug use in a nursing home: a 6-year retrospective. J Appl Gerontol. 2003;22(4):474-89.

13. Nygaard HA, Naik M. Use of psychotropic drugs in homes for the aged in Bergen, Norway: a comparative study. Norwegian J Epidemiol. 1998;8(2):133-8.

14. Snowdon J. A follow-up survey of psychotropic drug use in Sydney nursing homes. Med J Aust. 1999;170:299-301.
15. Lakey SL, Gray SL, Sales AE, Sullivan J, Hedrick SC. Psychotropic use in community residential care facilities: a prospective cohort study. Am J Geriatr Pharmacother. 2006;4(3):227-35.

16. Briesacher BA, Limcangco R, Simoni-Wastila L, Doshi JA, Levens SR, Shea $\mathrm{DG}$, et al. The quality of antipsychotic drug prescribing in nursing homes. Arch Intern Med. 2005;165:1280-5.

17. Rochon PA, Stukel TA, Bronskill SE, Gomes T, Sykora K, Wodchis WP, et al. Variation in nursing home antipsychotic prescribing rates. Arch Intern Med. 2007;167(7):676-83.

18. Liperoti R, Mor V, Lapane KL, Pedone C, Gambassi G, Bernabei R. The use of atypical antipsychotics in nursing homes. J Clin Psychiatry. 2003;64(9):1106-12.

19. Shorr RI, Fought RL, Ray WA. Changes in antipsychotic drug use in nursing homes during implementation of the OBRA-87 regulations. JAMA. 1994;5:358-62.

20. Pitkala KH, Laurila JV, Strandberg TE, Tilvis RS. Behavioral symptoms and the administration of psychotropic drugs to aged patients with dementia in nursing homes and in acute geriatric wards. Int Psychogeriatr. 2004;16:61-74.

21. Snowdon J, Day S, Baker W. Why and how antipsychotic drugs are used in 40 Sydney nursing homes. Int J Geriatr Psychiatry. 2005;20(12):1146-52.

22. Siqueira GR, Vasconcelos DT, Duarte GC, Arruda IC, Costa JAS, Cardoso RO. Análise da sintomatologia depressiva nos moradores do Abrigo Cristo Redentor através da aplicação da Escala de Depressão Geriátrica (EDG). Cienc Saude Coletiva. 2009;14(1):253-9.

23. Oliveira CAA, Lima SA. Idoso em movimento: proposta para melhor qualidade de vida na velhice [monografia]. Recife: Universidade Federal de Pernambuco; 2001. 68 f.

24. Fick DM, Cooper JW, Wade WE, Waller JL, Maclean JR, Beers MH. Updating the Beers criteria for potentially inappropriate medication use in older adults: results of a US consensus panel of experts. Arch Intern Med. 2003;163:2716-24.

25. Schnelle JF, Ouslander JG, Simmons SF, Alessi CA, Gravel MD. The nighttime environment, incontinence care, and sleep disruption in nursing homes. J Am Geriatr Soc. 1993;41(9):910-4.

26. Kim H, Whall AL. Factors associated with psychotropic drug usage among nursing home residents with dementia. Nurs Res. 2006;55(4):252-8.

27. Loyola Filho AI, Uchoa E, Firmo JOA, Lima-Costa MF. Influência da renda na associação entre disfunção cognitiva e polifarmácia: Projeto Bambuí. Rev Saude Publica. 2008;42(1):89-99.

28. Wortmann AC, Grüdtner MC, Fialho AF, Jardim Neto JC, Schaefer LG, Sehn F, et al. O consumo de benzodiazepínicos em Porto Alegre. Rev Ass Med Bras. 1994;40(4):265-70.

29. Linden M, Lecrubier Y, Bellantuono C, Benkert O, Kisely S, Simon G. The prescribing of psychotropic drugs by primary care physicians: an international collaborative study. J Clin Psychopharmacol. 1999;19(2):132-40.

30. Linjakumpu T, Hartikainen S, Klaukka T, Koponen H, Kivela SL, Isoaho R. Psychotropics among the home-dwelling elderly - increasing trends. Int J Geriatr Psychiatry. 2002;17(9):874-83.

31. Holmquist IB, Svensson B, Hoglund P. Psychotropic drugs in nursing- and oldage homes: relationships between needs of care and mental health status. Eur J Clin Pharmacol. 2003;59(8-9):669-76.

32. Hohmann AA. Gender bias in psychotropic drug prescribing in primary care. Med Care. 1989;27(5):478-90.

33. Ott BR, Lapane KL, Gambassi G. Gender differences in the treatment of behavior problems in Alzheimer's disease. Neurology. 2000;54:427-32.

34. Lindesay J, Matthews R, Jagger C. Factors associated with antipsychotic drug use in residential care: changes between 1990 and 1997. Int J Geriatr Psychiatry. 2003;18(6):511-9. 